\title{
Deep-towed high resolution seismic imaging II: Determination of P-wave velocity distribution
}

\author{
Marsset Bruno ${ }^{1,{ }^{*}}$, Ker Stephan ${ }^{1}$, Thomas Yannick ${ }^{1}$, Colin Florent ${ }^{1}$ \\ ${ }^{1}$ Ifremer, REM/GM/LAD, Centre de Brest, BP 70, 29280 Plouzane, France \\ * Corresponding author : Bruno Marsset, email address : Bruno.Marsset@ifremer.fr
}

\begin{abstract}
:
The acquisition of high resolution seismic data in deep waters requires the development of deep towed seismic sources and receivers able to deal with the high hydrostatic pressure environment. The low frequency piezoelectric transducer of the SYSIF (SYstème Sismique Fond) deep towed seismic device comply with the former requirement taking advantage of the coupling of a mechanical resonance (Janus driver) and a fluid resonance (Helmholtz cavity) to produce a large frequency bandwidth acoustic signal $(220-1050 \mathrm{~Hz})$. The ability to perform deep towed multichannel seismic imaging with SYSIF was demonstrated in 2014, yet, the ability to determine P-wave velocity distribution wasn't achieved. P-wave velocity analysis relies on the ratio between the source-receiver offset range and the depth of the seismic reflectors, thus towing the seismic source and receivers closer to the sea bed will provide a better geometry for P-wave velocity determination. Yet, technical issues, related to the acoustic source directivity, arise for this approach in the particular framework of piezoelectric sources. A signal processing sequence is therefore added to the initial processing flow. Data acquisition took place during the GHASS (Gas Hydrates, fluid Activities and Sediment deformations in the western Black Sea) cruise in the Romanian waters of the Black Sea. The results of the imaging imagingimaiaging processing are presented for two seismic data sets acquired over gas hydrates and gas bearing sediments. The improvement in the final seismic resolution demonstrates the validity of the velocity model.
\end{abstract}

\section{Highlights}

- Perform deep towed High Resolution velocity determination. I Increase seismic imaging performance. Bottom simulating Reflecto.

Keywords : Deep-towed seismic, seismic imaging, P-wave velocity distribution 
High Resolution (220-1050 Hz) seismic acquisition performed in great water depth using deep-towed systems provides unrivalled lateral resolution when compared to conventional surface seismic methods $(50-250 \mathrm{~Hz}$, Chapman et al., 2002; Gettrust et al., 2004). This gain in lateral resolution is primarily related to the acquisition geometry as it decreases the width of the first Fresnel zone. The gain also depends on the processing sequence in which imaging algorithms (migration) improve the resolution down to the mean wavelength of the seismic signal. Yet this ideal resolution may only be obtained if the correct velocity model is used. This requires the determination of the fine scale P-wave velocity distribution.

In a general case, P-wave velocities in shallow marine sediments are not expected to display strong variations due to the relative homogeneity of the medium. However, in the presence of existing fluid systems including the existence of gas or/and gas hydrates, the P-wave velocity distribution can vary significantly both laterally and vertically, making quantifying the velocity distribution (e.g., Helgerud et al, 1999) a key factor in gas/gas hydrate saturation assessment (He et al, 2009).

A large number of methods are available to access to the seismic velocity distribution. These methods differ according to their initial hypothesis, from the simplest (flat horizontal reflectors, Pythagorean theorem) to the most complex (calculation of the Green functions in a complex medium), yet they all rest on the accuracy of the source-receiver positions which remains the key point of any seismic acquisition. successfully determined P-wave velocity distribution and thus acoustically characterized the medium (Wood et al., 2003, 2008). Nevertheless, the DTAGS pre-stack processing approach rests on the use of "Super Gather", up to $300 \mathrm{~m}$-large and therefore the resulting smearing of information limits the accuracy for the determination of fine-scale P-wave velocity models.

54 A digital deep-towed multichannel streamer (52 traces@2m) has been developed and the feasibility of performing high-resolution (220-1050 Hz) multichannel seismic imaging in deep water has been demonstrated (Marsset et al., 2014). Yet, the seismic data acquired during early sea trials of this streamer did not allow to determine the P-wave velocity distribution as the altitude of the towed fish was kept conservatively high at 150 $\mathrm{m}$ above the sea floor.

59 One of the objectives of the GHASS project aims at studying the dynamics of gas hydrates and free gas 60 associated with geological and climate processes (Ker et al., 2015). The GHASS project rests on a 61 multidisciplinary approach including the fields of geotechnics, sedimentology and geochemistry. Such precise, 
but local, measurements require in turn high resolution spatial information to correlate them. The deep towed seismic source SYSIF (Marsset et al., 2010), together with its digital multichannel streamer (Marsset et al., 2014), was therefore deployed to obtain high resolution seismic image as well as fine-scale P-wave velocity model.

The deep towed high resolution seismic source SYSIF (Marsset et al., 2010 ; Ker et al., 2010) and its digital multichannel streamer have already been described as well as the processing sequence of deep towed multichannel seismic data (Marsset et al., 2014). This contribution focuses on the determination of P-wave velocity distribution of gas and gas hydrates bearing sediments, highlighting the specific acquisition constraints and the dedicated processing sequence to get full benefit of pre-stack depth migration - velocity model building seismic imaging scheme .

\section{METHODOLOGY}

\section{A. $\quad$ Acoustic source directivity}

The ability to determine P-wave velocities from multichannel seismic data depends on the ratio between the source-receiver offset range and the depth of the seismic reflectors, i.e. a function of the incidence angle. In the framework of deep towed acquisition, this ratio can be easily expanded by lowering the system closer to the sea floor : the lower the altitude over the sea bottom, the larger the interval of incidence angles. For safety purposes considering the overall streamer length of $120 \mathrm{~m}$, the minimum altitude with respect to the sea-bed is set to $50 \mathrm{~m}$. Such a geometry allows to record incidence angles exceeding $45^{\circ}$ for the upper sedimentary layers. Yet, one technical issue related to the acoustic source directivity arises for this approach in the particular framework of piezoelectric seismic sources.

The large frequency bandwidth of the JH220-6000 Janus-Helmholtz acoustic source (220-1050 Hz) is obtained by coupling of a mechanical resonance (Janus driver) and a fluid resonance (Helmholtz cavity) (Le Gall, 1999). Each transmitter has its own directivity, and the coupling between the two resonances results in a specific directivity for the transducer. The directivity of the JH220-6000 has been modeled using the ATILA software (figure 1), a finite element software designed for the analysis of mechanical 2D/3D structures hosting piezoelectric materials (Hennion et al., 1990 ; Le Gall \& Marsset, 2007). Based on acoustic measurements acquired using OBH's (Ocean Bottom Hydrophone), Ker et al (2010) evaluated the main lobe of reflectivity of the JH220-6000 to 40 (@ -3dB), nevertheless the strong attenuation of the central frequencies ( e.g. -8 dB @ $800 \mathrm{~Hz} @ 30^{\circ}$, figure 1) observed on the ATILA simulations suggests that the useful aperture is limited to $30^{\circ}$. 


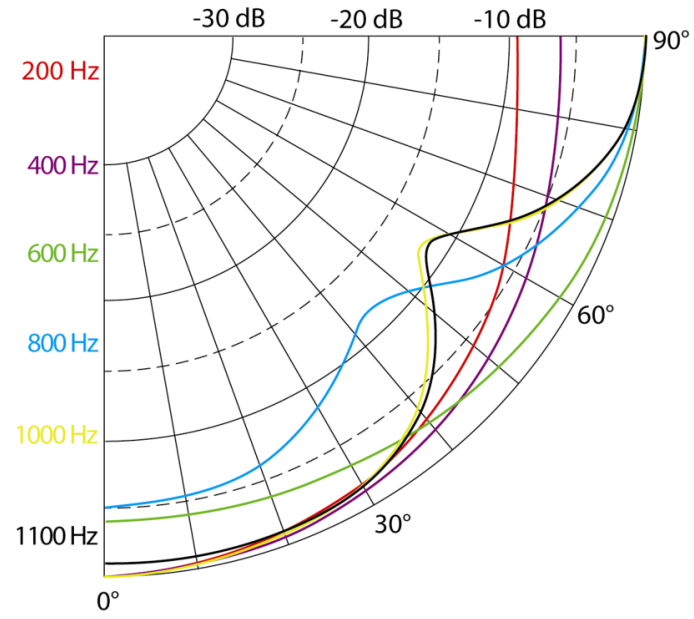

Fig. 1. : Relative directivity of the JH220-6000 piezoelectric transducer performed using ATILA software. $0^{\circ} / 90^{\circ}$ corresponds respectively to the vertical axis and to the horizontal axis of the transducer.

The electric pilot source signal is a 100 milliseconds Linear FM (Linear Frequency Modulation) signal ranging from 220 to $1050 \mathrm{~Hz}$. This initial signal is amplitude modulated taking into account the TVR (Transmit Voltage Response) of the transducer, in order to obtain an almost flat acoustic signal with a constant SL (Sound Level) of $196 \mathrm{~dB}$ ref $1 \mu \mathrm{Pa} @ 1 \mathrm{~m}$ over the entire frequency bandwidth in the nadir of the transducer. The "vertical" far field acoustic signature has been previously recorded (Ker et al., 2010). The "horizontal" far-field signature was recorded on the far hydrophone (in open water) during the GHASS cruise in order to assess the impact of the directivity of the transducer on the acoustic signal (figure 2, a-b). The classic processing sequence of linear FM signals involves performing either matching filter or deconvolution of the raw data with a known template in order to maximize the temporal resolution. The results of the deconvolution of the two signatures using the "vertical" signature as template clearly points out the incompatibility of this classic processing sequence for seismic data acquired with Janus-Helmholtz-like transducers as the source signature depends on the incidence angle (figure 2, c-d). 

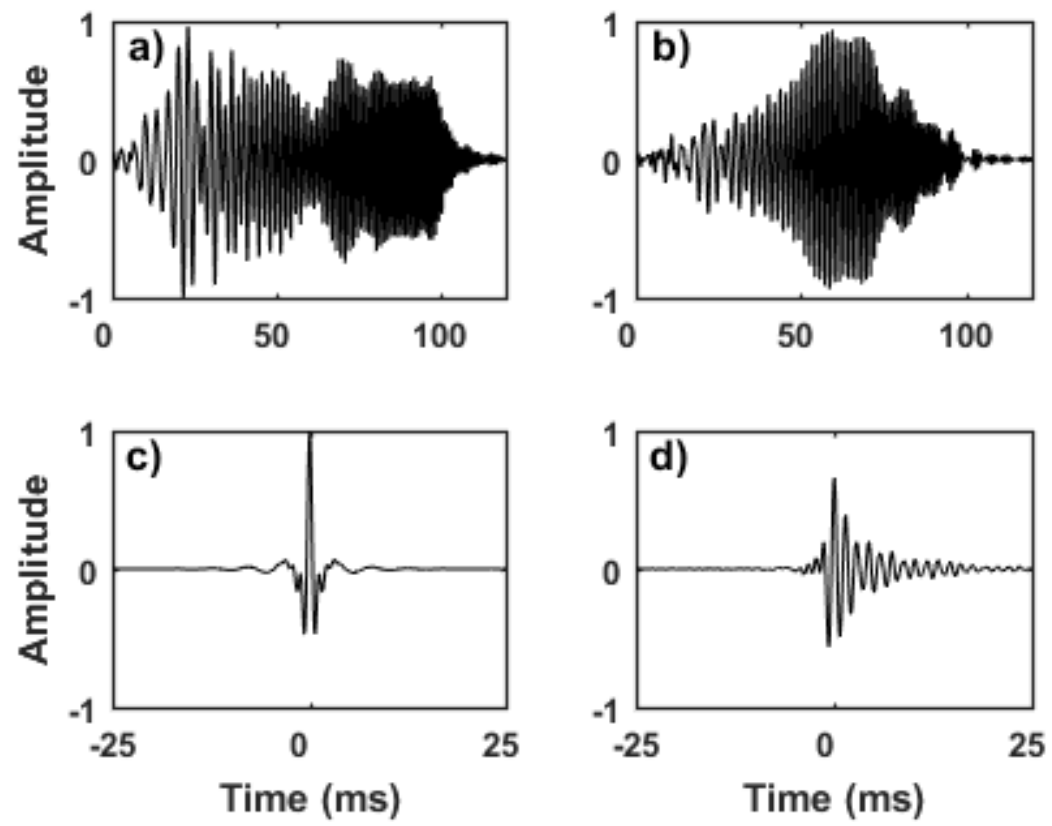

111 Fig. 2. : "Vertical" (a) and "horizontal" (b) acoustic signatures of the JH220-6000 transducer.

112 c): Result of the deconvolution of the "vertical" signature (a) with the "vertical" signature (a).

113 d) : Result of the deconvolution of the "horizontal" signature (b) with the "vertical" signature (a)

\section{B. Acoustic interferences}

117 The zero offset travel time corresponding to the source/sea-bottom/receiver ray is to be $66 \mathrm{~ms}$ for an altitude of

11850 meters (@ $1500 \mathrm{~m} / \mathrm{s}$ ) and therefore this ray will interfere with the direct arrival corresponding to the source/receiver ray. A processing step was therefore added, prior to source deconvolution, using STFT (short

120 term Fourier transform) which allows to filter data in the Time-Frequency domain, a domain well adapted to

121 Linear FM type signals (Allen, 1977).

122 The method was first applied on synthetic data (figure 3). The synthetic seismogram consists in the combination

123 of a zero-delay "horizontal" signature with a 66 milliseconds delayed / $20 \mathrm{~dB}$ attenuation "vertical" signature

124 thus mimicking a reflection on the sea bed with a 0.1 reflection coefficient for a monostatic acquisition. The

125 synthetic seismogram is then transformed, taking advantage of STFT algorithm, in the time-trequency domain

126 where the two signatures appear as separated signals. The modulus of the STFT corresponding to the direct

127 arrival, i.e. "horizontal" signature, is then zeroed and the inverse STFT is performed to compute the processed

128 seismogram back into the time domain. After deconvolution, the gain in $\mathrm{S} / \mathrm{N}$ (signal to noise ratio) is evaluated

129 to $18 \mathrm{~dB}$ on synthetic data. 
a)

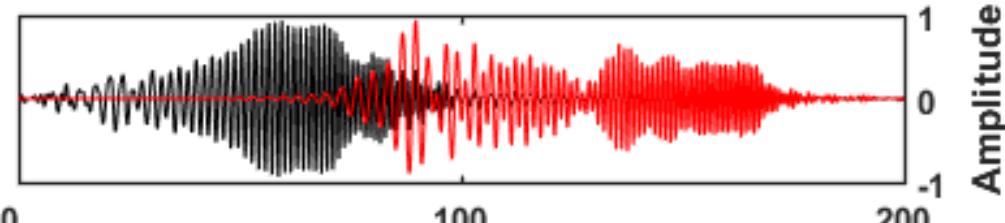

b)

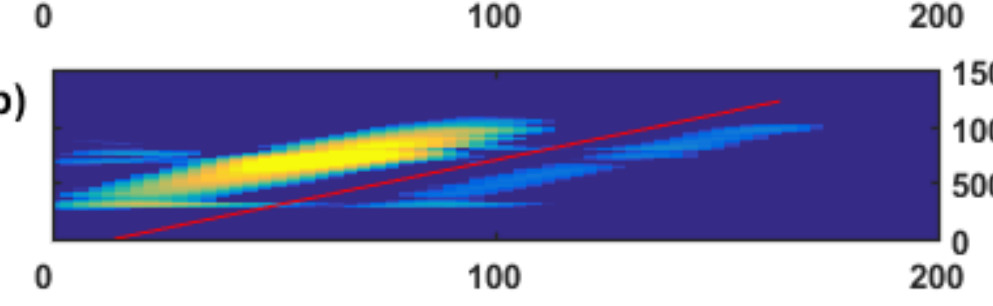

c)

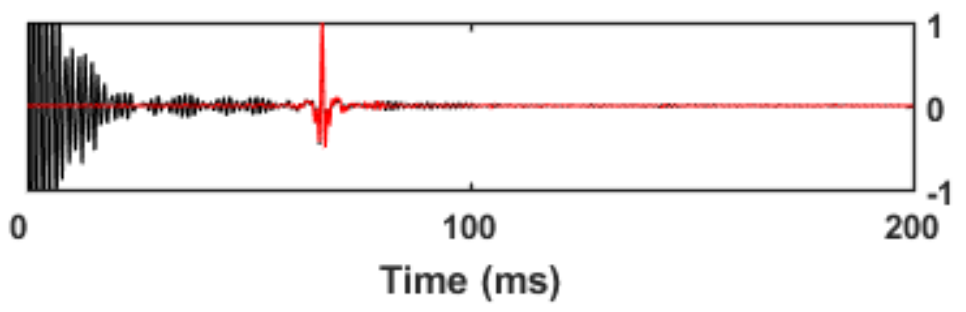

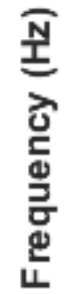

$\frac{\text { 믈 }}{\frac{\text { 튼 }}{4}}$

Fig. 3. : Signal processing sequence

132 a): Raw synthetic seismogram (black/red curves without/with time-frequency filtering), data are normalized.

133 b) : STFT modulus of the synthetic seismograms, data left of the red line are muted. The red line corresponds to 134 the slope of the linear frequency modulated signal.

135 c) : Synthetic seismogram after deconvolution (black/red curves without/with time-frequency filtering), data are normalized.

138 The former signal processing sequence was then applied on the SYSIF seismic data. Data are first deconvolved

139 using the "horizontal" acoustic signature in order to precisely pick the direct arrivals (i.e. source-receiver ray) for

140 each hydrophone. Raw traces are then time shifted to the direct arrival time of the first receiver so that filtering

141 can use the same mask in the time-frequency domain for the entire data set. After filtering, data are shifted back

142 to their original time and deconvolved with the proper template, i.e. "vertical" acoustic signature. The results are

143 presented on figure 4 which illustrates the gain in $\mathrm{S} / \mathrm{N}$. 
a)

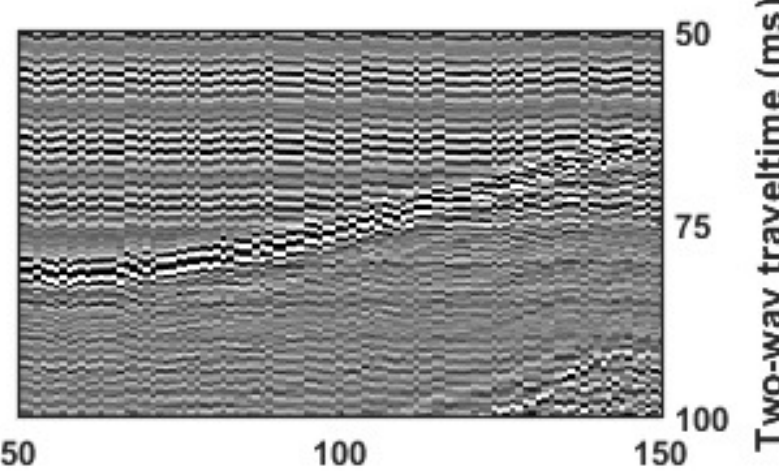

b)

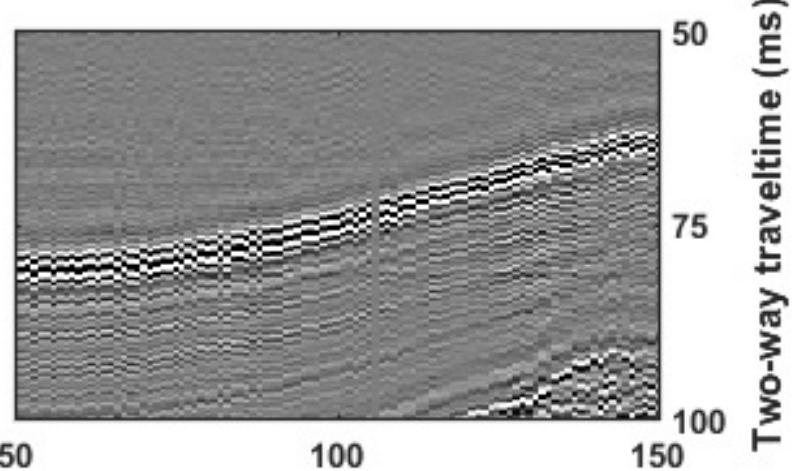

Shot number

144
145

146

147

148

149

150 Positioning is the key issue in seismic imaging processing where the calculation of travel times rests on the

151 positions of the different source-receiver pairs. A relative error of one wavelength, i.e. $\approx 2 \mathrm{~m}$ for the mean wavelength of the JH220-6000 transducer, will prevent a proper focalization of the seismic events and therefore

153 will lower the optimal resolution.

154 The streamer geometry is evaluated based on the hydrophones MEMS (Micro-Electro-Mechanical System) pitch

155 and heading values. The geographic location of the source is ensured by an acoustic USBL (ultra short base line)

156 (Marsset et al., 2014).

157 In order to evaluate the positioning accuracy, the theoretical sea bed reflection travel times were calculated using

158 the streamer geometry together with a $10 \mathrm{~m}$ DTM (digital terrain model), obtained from a multibeam survey, in a

159 2D ray tracing in-house algorithm. The sea floor reflected travel times were picked on a CRG (common receiver

160 gather) basis for all shots. The time differences between modeled and observed (picked) travel times for each

161 hydrophone reveal a Gaussian distribution which allows the standard deviation to be evaluated after curve 


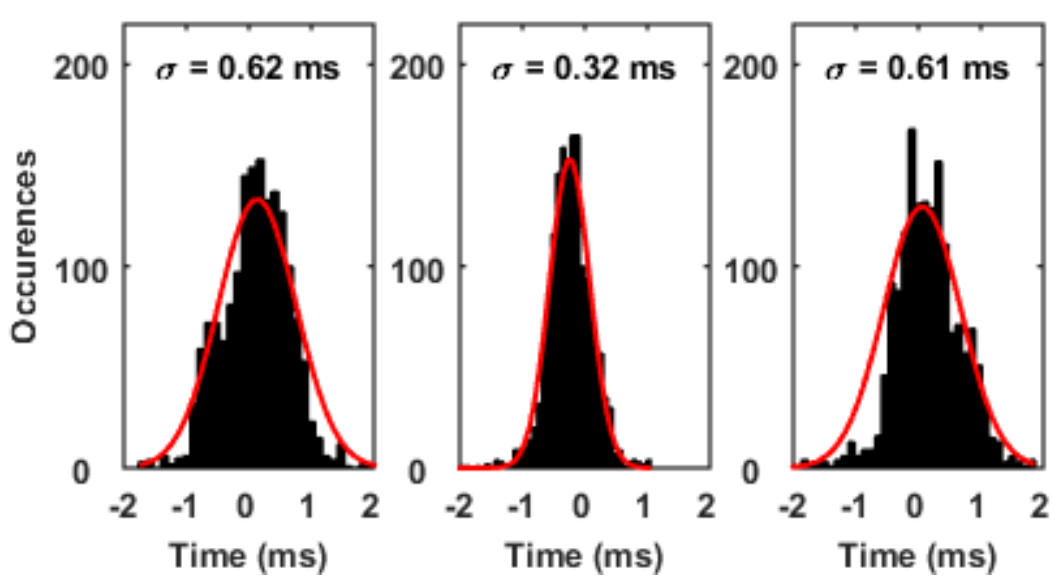

Fig. 5. : Distributions of the sea floor reflected arrival time differences between modeled and observed (picked) values. From left to right, hydrophones $n^{\circ} 1,26$ and 52 (line GHASS PLO1PRO2B).

\section{VELOCITY DETERMINATION}

The particular geometry of the acquisition where the source receiver geometry is perpetually changing, does not allow the seismic data to be processed in a "conventional" marine sequence: CMP (common mid point) sorting (NMO) normal move out - stack (Asakawa et al., 2009). We adapted a pre-stack processing approach in order to take into account the real $(\mathrm{X}, \mathrm{Y}, \mathrm{Z})$ positions of both sources and receivers in the imaging process.

The imaging process applies Kirchhoff depth migration to each CRG. The Green functions are calculated using the finite difference Eikonal equation solver from Podvin and Lecomte (1991). The source and receiver travel times are computed using $0.1 \mathrm{~m} * 0.1 \mathrm{~m}$ grid cells in order to take into account the high frequency content of the acoustic signal. The migrated traces are calculated every 1.0 meters which satisfy the aliasing limits for the JH220-6000 frequency bandwidth. The migration aperture is limited to $30^{\circ}$ according to the directivity of the transducer.

The different migrated sections are then sorted into CIG (Common Image Gathers), i.e. to build an image of the same terrain location obtained from the different receivers. If the migrated traces of a CIG are identical for the different receivers, i.e. flat reflections along the offsets, this shows the validity of the velocity model. This approach is carried out in a layer-stripping iterative scheme to obtain, simultaneously, the variation of P-wave velocity with depth and the imaging result. 
186 The selection of the "correct" velocity is presented through a simplified approach (migration velocity scan).

187 Figure 6 presents a CIG migrated using different velocities. A number of selected horizons are picked.

188
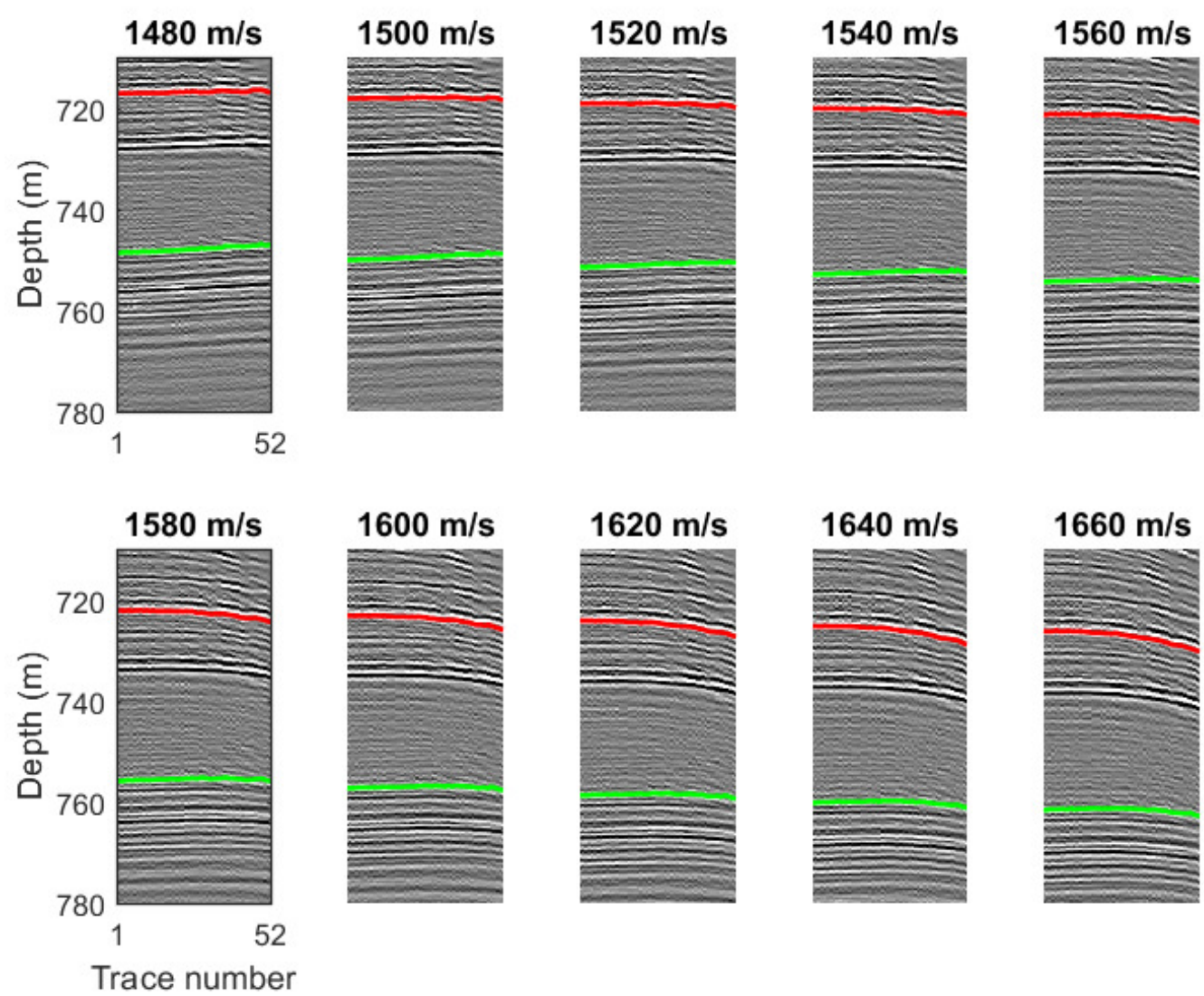

190 Fig. 6. : Migration velocity scan. An identical CIG obtained from different constant velocity migration. Selected

191 horizons are picked.

192

193 The different horizons are then fitted with a second order polynomial. The first coefficient of the polynomial

194 accounts for the "curvature", the second coefficient for the "linear trend". The correct velocity is selected where

195 both the "curvature" and the "linear trend are reduced to zero (figure 7).

196

197 

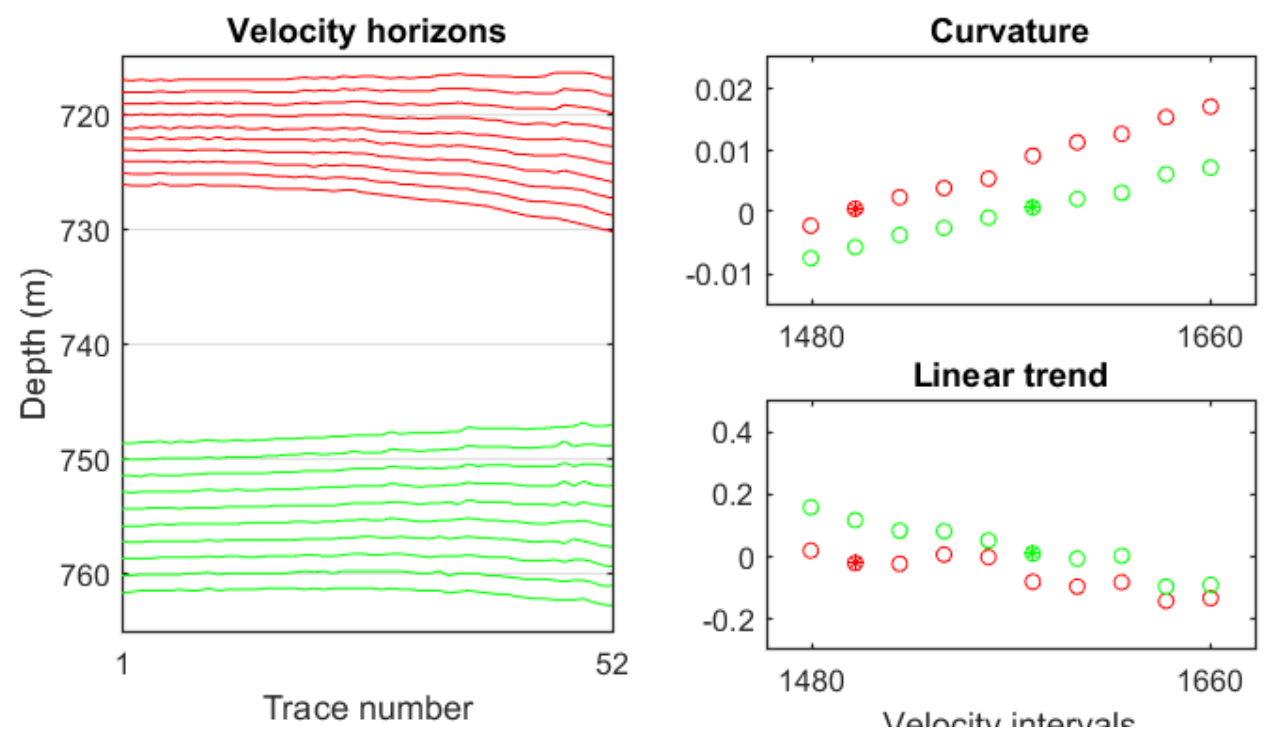

Trace number

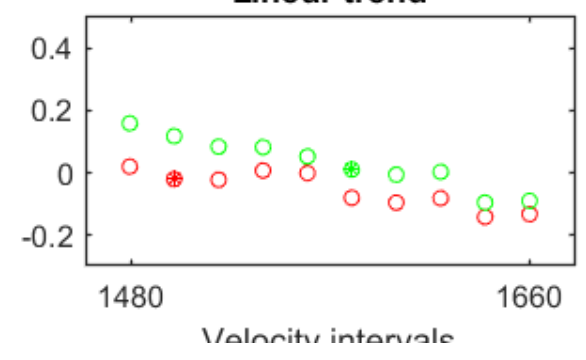

Fig. 7. : The selected velocity horizons are fitted with a second order polynomial. The correct velocity is selected where the "curvature" (1st polynomial coefficient) and the "linear trend" (2nd polynomial coefficient) are both reduced to zero (2nd velocity $(1500 \mathrm{~m} / \mathrm{s})$ for the upper (red) layer, 6th velocity $(1580 \mathrm{~m} / \mathrm{s})$ for the lower green) layer). hours, 30 profiles) at an average speed of 2 knots (Ker et al., 2015). The altitude of the towed fish over the sea 209 bottom was set to 100 meters in most cases in order to avoid interferences between direct and reflected arrivals. Two of the profiles were re-acquired with an altitude of 50 meters over the sea bed in order to better constrain

211 the calculation of P-wave velocity.

214 The line was first acquired with an altitude of 100 meters (line PL01PR02). On board processing of this line 215 underlines the presence of strong amplitude anomalies interpreted as a BSR (Bottom Simulating Reflector), a 216 reflector which mimics the seabed reflection and which is presumably caused by gas hydrates bearing sediments overlying gassy sediments. As the presence of gas hydrates is likely to cause an increase in the P-wave velocity

218 distribution, the line was re-acquired with an altitude of 50 meters and a shooting rate of one per 2.2 seconds. 


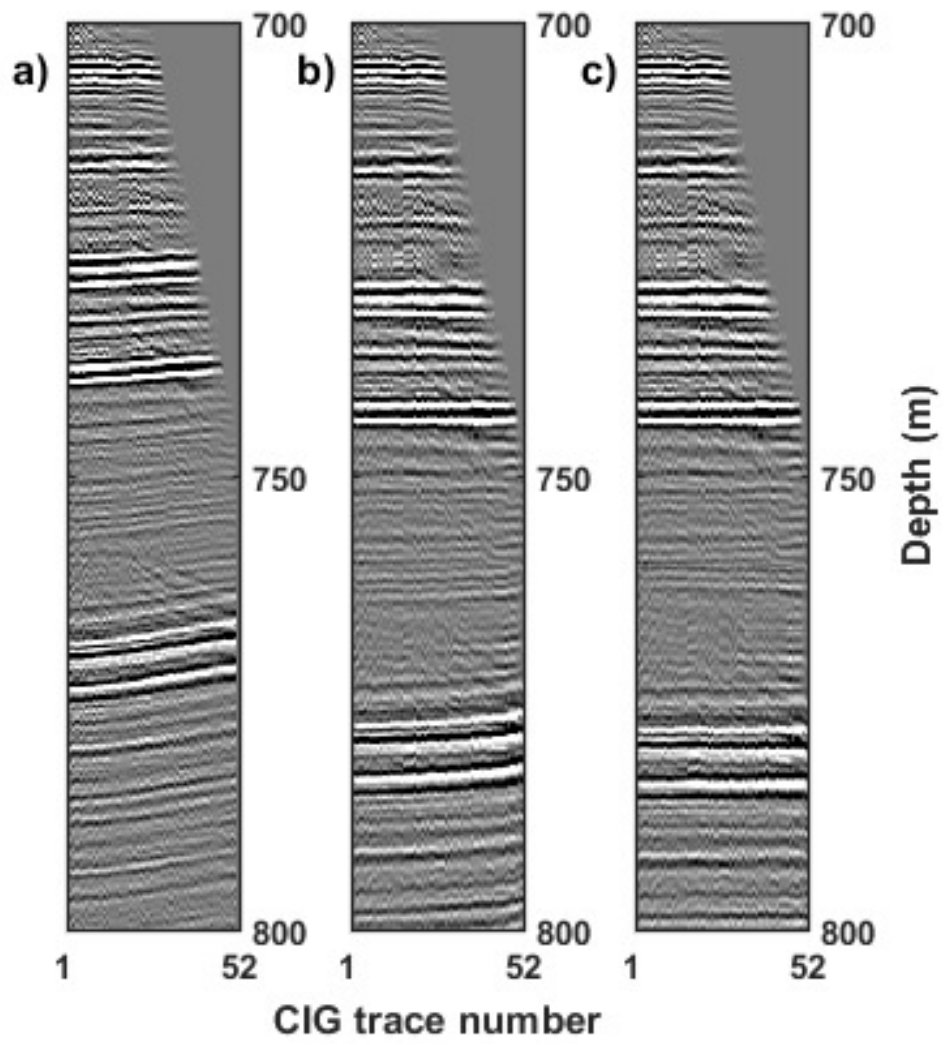

220 Fig. 8. : Common Image Gather (line PL01PR02B)

221 a): One layer velocity model: Water velocity $(1480 \mathrm{~m} / \mathrm{s})$

222 b) : Two layers velocity model:1480m/s - 1700 m/s@ 720 meters

223 c) : Three layers velocity model:1480m/s - 1700 m/s@720 meters-1800 m/s@775 meters

225 The first iteration of the velocity analysis was performed with a constant (water) velocity of $1480 \mathrm{~m} / \mathrm{s}$ derived

226 from XCTD (expendable conductivity-temperature-depth) probe measurements (figure 8a). The seabed

227 reflection appears flat on the CIG while deeper reflectors displays an upward curvature, characteristic of an

228 increase of the P-wave velocity. The second iteration was performed using a two layers velocity model (figure

229 8b) with a $1700 \mathrm{~m} / \mathrm{s}$ value. Eventually the third iteration used a three layers velocity model, adding an $1800 \mathrm{~m} / \mathrm{s}$

230 layer down to the BSR (figure 8c).

231 The resolution enhancement associated to the velocity model in the final imagery result is obvious (figure 9a).

232 The figure presents a comparison of the imagery results for the constant (water) velocity model (figure 9b) and

233 the three layers velocity model (figure 9c), where the latter displays well focussed reflectors as well as sharp

234 terminations of the amplitude anomalies. 


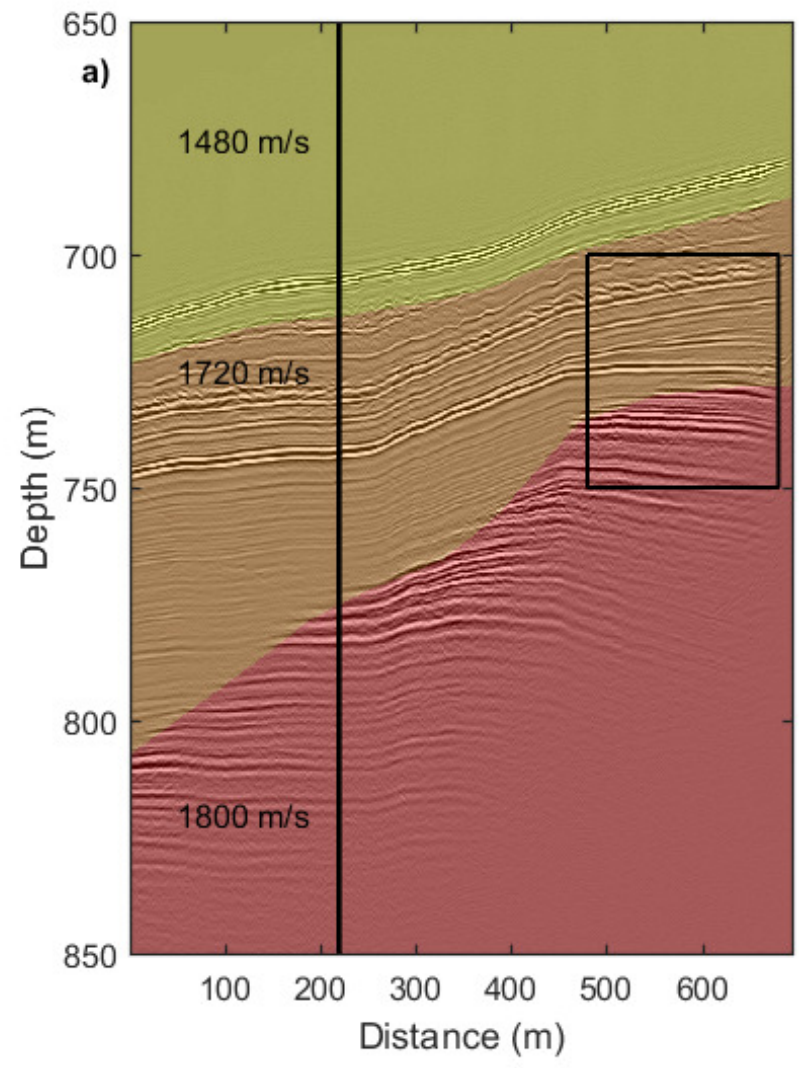

b)

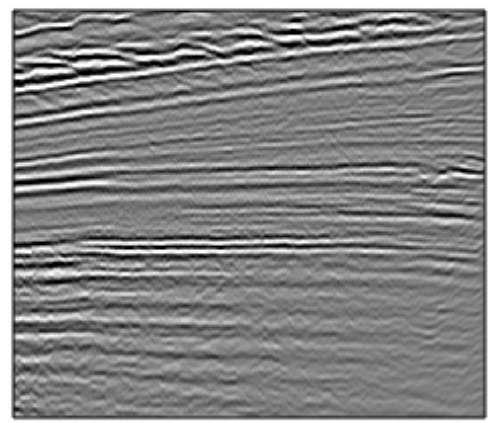

c)

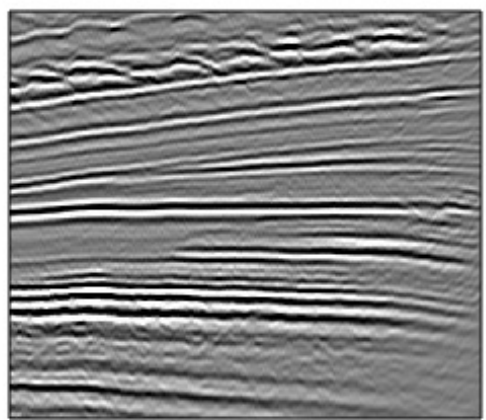

Fig. 9. : Imaging performance improvement

a): Regional line calculated with the three layers velocity model (green), the vertical red line corresponds to the position of CIG 210 of Fig. 8. The velocity model is superimposed.

b) : Imaging results of the red inset for the constant (water) velocity model

c) : Imaging results of the red inset for the three layers velocity model

\section{$242 \quad$ B. $\quad$ Line PLO3PRO6}

244 During the GHASS cruise, the water column was thoroughly monitored using multibeam technology with water 245 columns record, in order to map possible offshore seeps (e.g. Dupre et al., 2015). The deep towed seismic line 246 PL03PR06 was acquired over a major gas seep observed in the water column suggesting the presence of free gas 247 in the sedimentary column (acquisition parameters : altitude $=50 \mathrm{~m}$, shooting rate : one per 2.5 seconds). 


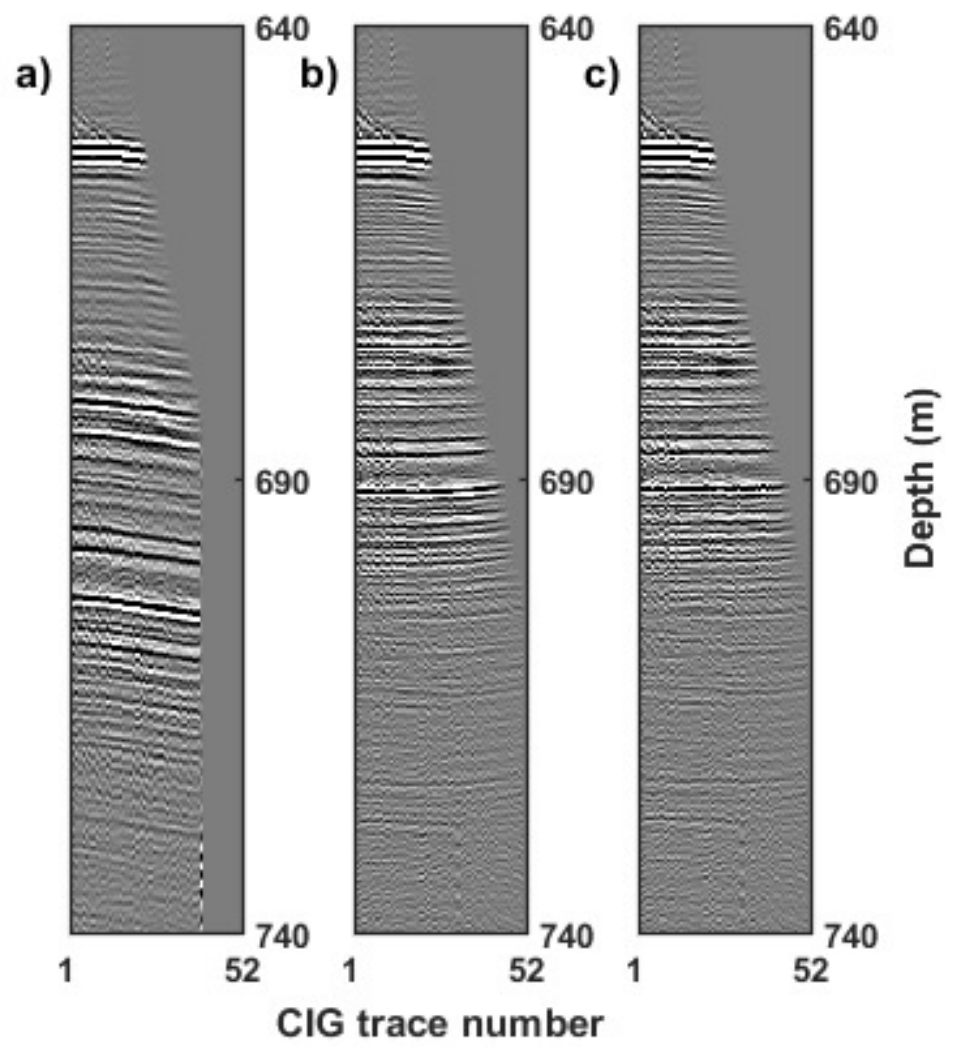

249 Fig. 10. : Common Image Gather

250 a): One layer velocity model: Water velocity $(1480 \mathrm{~m} / \mathrm{s})$

251 b) : Two layer velocity model: $1480 \mathrm{~m} / \mathrm{s}-1100 \mathrm{~m} / \mathrm{s} @ 665$ meters

c): Three layer velocity model:1480m/s-1100 m/s@665 meters-1250 m/s @680 meters

254 The first iteration of the velocity analysis was performed with a constant (water) velocity of $1480 \mathrm{~m} / \mathrm{s}$ obtained

255 from XCTD probing (figure 10a). The seabed reflection appears nearly flat on the CIG while deeper reflectors

256 displays downward curvature, characteristic of a decrease of the P-wave velocity. The second iteration was

257 performed with a two layers velocity model (figure 10b) with an $1100 \mathrm{~m} / \mathrm{s}$ value. This model allows to flatten

258 reflectors down to 680 meters but tends to overcorrect underlying reflectors, therefore, the third iteration uses a

259 three layers velocity model, adding an $1200 \mathrm{~m} / \mathrm{s}$ layer (figure 10c).

261 Again the gain in resolution associated to the velocity model in the final imagery result is clearly visible (figure

262 11a). The figure presents a comparison of the imagery results for the constant (water) velocity model (figure

$26311 \mathrm{~b}$ ) and the three layers velocity model (figure 11c), where the latter displays well focussed reflectors. 


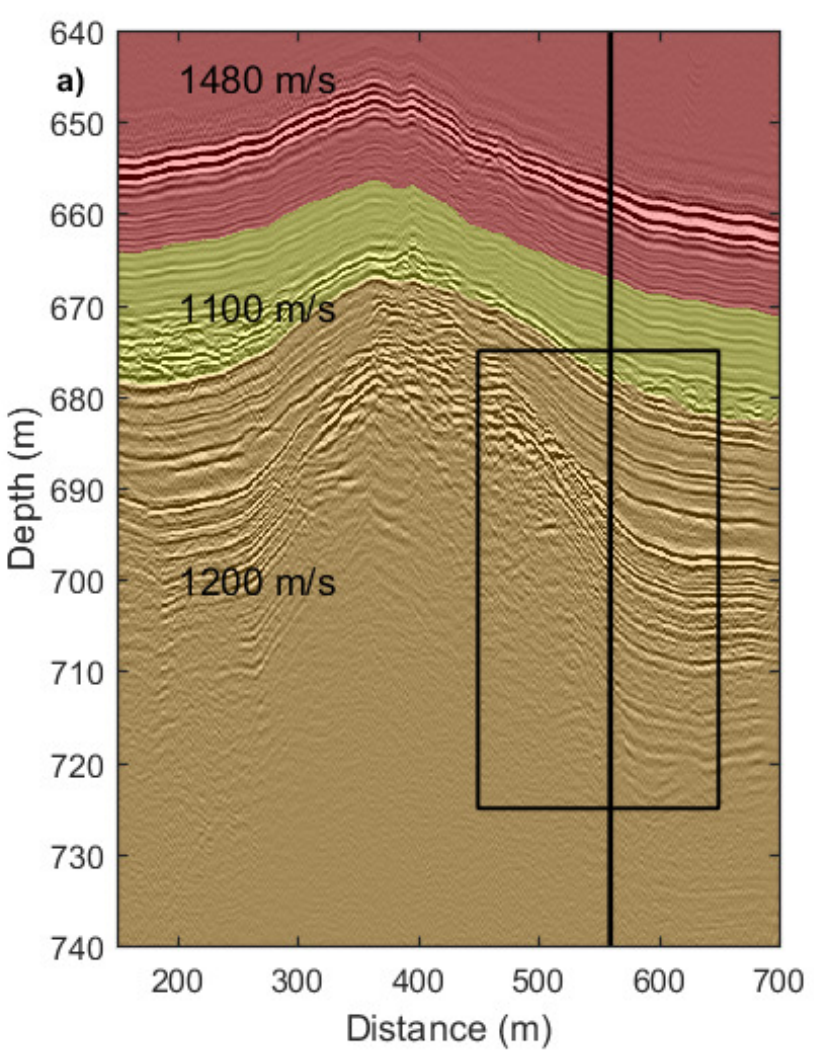

b)

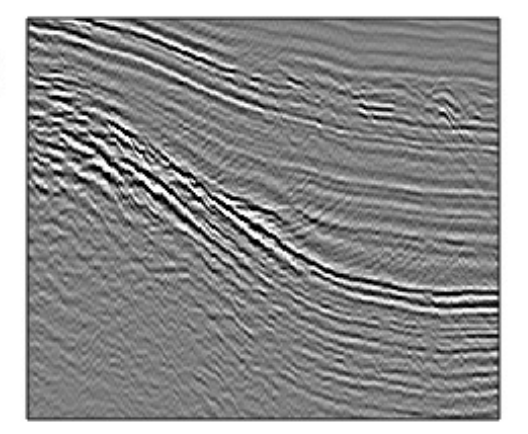

c)



Fig. 11. : Imaging performance improvement

a) : Regional line calculated with the three layers velocity model (green), the vertical red line corresponds to the position of 560CIG of Fig. 10. The velocity model is superimposed.

b) : Imaging results of the red inset for the constant (water) velocity model

c) : Imaging results of the red inset for the three layers velocity model

\section{DISCUSSION}

The impact of the P-wave velocity distribution on the final vertical resolution was tentatively quantified on line

274 PL03PR06. The average wavenumber spectrum of a selected part of the line for the constant and the three layers velocity models (figure 12a) are calculated (figure $12 \mathrm{~b}$ ). As a result, the central wavenumber is shifted from $2 / 3$ $\mathrm{m}^{-1}(1.5 \mathrm{~m})$ for the water velocity model to $1 \mathrm{~m}^{-1}(1 \mathrm{~m})$ for the three layers velocity model.

278 An evaluation of the local gain in amplitude was performed on line PL01PR02B where reflectors were picked on 279 the imaging results of the constant and three layers velocity model (figure $10 \mathrm{c}-\mathrm{d}$ ). The average gain is evaluated 280 to $7 \mathrm{~dB}$ for this reflector. 
a)



c)

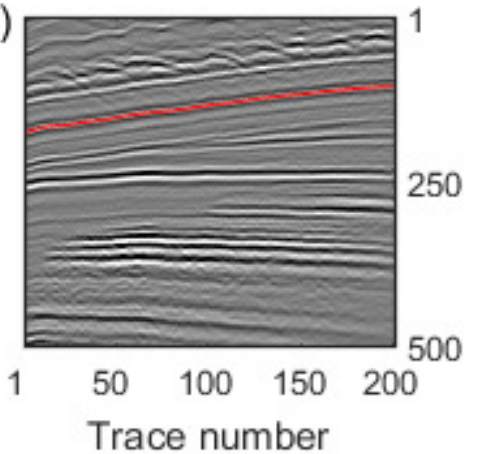

b)

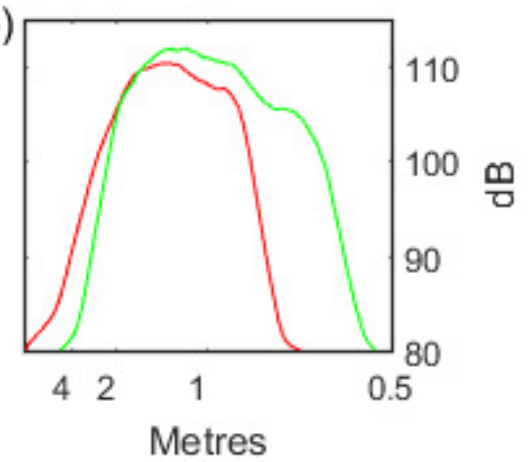

d)

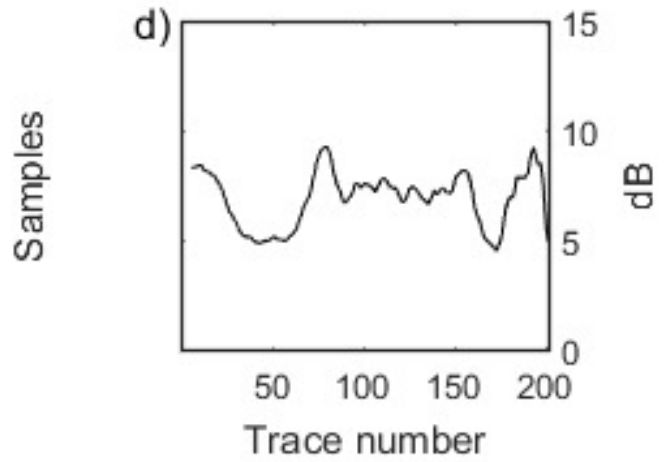

Fig. 12. : Imaging performance improvement

a) : Line PLO3PR06 (three layers velocity model) and b) : corresponding average wawenumber spectra (red: constant velocity model, green : three layers velocity model)

c) : Line PLO1PRO2B (three layers velocity model) and d) : amplitude ratio on a given reflector using constant and three layers velocity model.

In this contribution, we used Kirchhoff migration to perform seismic depth imaging of High Resolution deep

towed seismic data. Kirchhoff migration is an efficient algorithm, easy to implement with fast calculation times.

Nevertheless, this algorithm requires smooth variations of the velocity field and thus, in theory, it is not able to

cope with sharp interfaces as the seafloor interface or the gas hydrate / free gas interface. Migration methods

based on (FD) finite-difference migration are more suited to deal with fast space variation of the velocity field,

e.g. Ristow and Rühl, 1994, Plessix and Mulder, 2004 ; Brossier et al., 2008). These methods allows to define

very accurately the velocity field but have a very high computational cost. The algorithms associated to FD migration will be adapted to the specific deep-towed seismic geometry together with the development of antialiasing and over-sampling techniques in order to increase the resolution of the final velocity model.

\section{CONCLUSION}

The ability to perform high-resolution velocity analysis using a deep towed multichannel streamer has been demonstrated, based on the data set acquired during the GHASS cruise on the Romanian side of the Black sea. 
an increase of the velocities above a BSR (PL01PR02B) and 2) a decrease of these velocities associated with the presence of free gas assessed by acoustic flares in the water column (PL03PR06). The improvement in seismic imaging strongly supports the validity of the velocity results.

The GHASS project will be the first project to benefit from the spatial resolution achieved in deep towed High Resolution seismic imaging. The P-wave velocity distribution extracted from seismic data has now to be compared with in situ measurements obtained in the fields of geotechnics (velocity probe), sedimentology and geochemistry (gas nature/content). GHASS cruise data will then form a unique data set to understand the dynamics of gas hydrates and free gas in the geological and climate processes.

\section{Acknowledgments:}

The GHASS cruise was funded by French research institute for exploitation of the sea (Ifremer).

The development of the multichannel streamer was funded by Institut Carnot Ifremer EDROME.

The authors are indebted to the scientific party and to the crew of the GHASS cruise onboard French R/V "Pourquoi Pas ?".

\section{References:}

Allen, J. B., 1977. Short Time Spectral Analysis, Synthesis and Modification by Discrete Fourier Transform. IEEE Transactions on Acoustics, Speech and Signal Processing. ASSP-25 (3), 235-238.

Brossier, R., Virieux, J., and Operto, S. 2008, Parsimonious finite-volume frequency-domain method for 2D PSV-wave modeling, Geophysical JournalInternational, 175(2), 541-559

Chapman, N.R., J.F. Gettrust, R. Walia, D. Hannay, G.T. Spence, W.T. Wood and R.D. Hyndman, 2002, High Resolution deep-towed multichannel seismic survey of deep sea gas hydrates off western Canada, Geophysics, $67,1038-1047$

Dupré, S., C. Scalabrin, C. Grall, J. M. Augustin, P. Henry, A. M. C. Sengör, N. Görür, M. N. Cagatay, and L. Géli , 2015, Tectonic and sedimentary controls on widespread gas emissions in the Sea of Marmara: Results 
331 from systematic, shipborne multibeam echo sounder water column imaging, J. Geophys. Res. Solid Earth, 120(5), 2891-2912.

Gettrust, J.F., W. Wood, and S. Spychalski, 2004, High-resolution MCS in deepwater, The leading Edge, 23,

He, T., G. Spence, W. Wood, M. Riedel, and R. Hyndman, 2009, Imaging a hydrate-related cold vent offshore

Vancouver Island from deep-towed multichannel seismic data, Geophysics, 74(2), B23-B26.

Helgerud M. B., J. Dvorkin, and A. Nur, 1999, Elastic-wave velocity in marine sediments with gas hydrates :

Effective medium modeling, Geophysical Research Letters, 26(13), 2021-2024.

\section{2}

343 Hennion, A.C., Bossut, R., and Decarpigny, J.N., 1990, Analysis of the scattering of a plane acoustic wave by a

344 periodic elastic structure using the finite element method: Application to compliant tube gratings, Journal of the

Ker, S., B. Marsset, S. Garziglia, Y. Le Gonidec, D. Gibert, M. Voisset, and J. Adamy, 2010, High-resolution seismic imaging in deep sea from a joint deep-towed/OBH reflection experiment : application to a Mass

Ker S., V. Riboulot and Ghass Cruise Team, 2015, GHASS cruise report.

Le Gall Y., and B. Marsset, 2007, Développements électroacoustiques en sismique marine Très Haute

354 Résolution. Acoustique \& Techniques, (48), 17-22

Marsset B., E. Menut, S. Ker, Y. Thomas, J. P. Regnault, P. Leon, H. Martinossi, L. Artzner, D. Chenot, S. 
360 Marsset T., B. Marsset, S. Ker, Y. Thomas, and Y. Le Gall, 2010, High and very high resolution deep-towed 361 seismic system: Performance and examples from deep water geohazard studies, Deep-sea Research Part I362 oceanographic Research Papers, 57(4), 628-637.

363

364 Plessix, R.-E. and Mulder, W.A., 2004, Frequency-domain finite-difference amplitude-preserving migration, 365 Geophysical Journal International, 157(3), 957-987

366

367 Podvin P. and I. Lecomte, 1991, Finite difference computation of traveltimes in very contrasted velocity models.

368 A massively parallel approach and its associated tools, Geophysical Journal International, 105, 271-284.

369

370 Ristow D. and Rhül, T., 1994, Fourier finite difference migration, Geophysics, 55(4), 410421.

371

372 Wood, W., J. F. Gettrust, and S. E. Spychalski, 2003, A new deep-towed, multi-channel seismic system, Sea 373 Technology, 44(9), 44-49.

374

375 Wood, W. T., P. Hart, D. Hutchinson, N. Dutta, F. Snyder, R. Coffin, and J. F. Gettrust, J., 2008, Gas and gas 376 hydrate distribution around seafloor seeps in Mississipi Canyon, Northern Gulf of Mexico, using multi377 resolution seismic imagery, Marine and Petroleum Geology, 25(9), 952-959.

378

379

380 